\title{
The foraging perspective on economic choice
}

\author{
Benjamin Y. Hayden
}

Department of Neuroscience and

Center for Magnetic Resonance Research

University of Minnesota,

Minneapolis, MN 55455

\section{Correspondence:}

Benjamin Y. Hayden

CMRR Building,

University of Minnesota,

Minneapolis, MN 55455

Telephone: 425-749-2341

Email: benhayden@gmail.com

\section{Highlights}

- Foraging provides a basis for modeling economic choice based on adaptiveness

- Foraging choices are accept-reject; foraging models interpret binary choice accordingly

- The foraging view offers a different perspective on self-control decisions

- Economic and stopping decisions may have a common basis 


\begin{abstract}
Foraging theory offers an alternative foundation for understanding economic choice, one that sees economic choices as the outcome of psychological processes that evolved to help our ancestors search for food. Most of the choices encountered by foragers are between pursuing an encountered prey (accept) or ignoring it in favor of continued search (reject). Binary choices, which typically occur between simultaneously presented items, are special case, and are resolved through paired alternating accept-reject decisions limited by the narrow focus of attention. The foraging approach also holds out promise for helping to understand self-control and invites a reconceptualization of the mechanisms of binary choice, the relationship between choosing and stopping, and of the meaning of reward value.
\end{abstract}




\section{Introduction}

The need to pursue and consume food is a core part of any animal's behavioral repertoire and is a major driver of evolution. Decision-making is a key element of foraging - foragers must make tradeoffs that will maximize intake of desired prey and avoid unnecessary delay, risk, and effort. Foraging provides an approach to the psychology and neuroscience of economic decisionmaking, one that comes with a firm foundation, the principles of evolutionary adaptiveness (Pearson et al., 2014; Passingham and Wise, 2012). In this brief review, we provide a summary of the psychology and neuroscience of economic decision-making as seen through the lens of foraging.

\section{Foraging decisions are accept-reject decisions}

For most foragers, the distribution of prey in the environment is patchy in space and ephemeral in time (Stephens and Krebs, 1986). As foragers search, prey are generally encountered one at a time and the forager's decision is whether to pursue (accept) or ignore (reject) the prey item. This principle is true for both for a forager searching for individual prey organisms within a patch and foragers surveying multiple patches. The elemental decision in foraging, then, is the accept-reject decision and not, as in microeconomics, a binary choice between simultaneously presented items.

Although accept-reject decisions are ostensibly binary choices between two simultaneously appearing items (i.e. accept and reject), they function differently in practice. Pursuing a prey item is active, it leads to reward consumption (formally, it is exploitative), and it involves a change from the current state; capture normally triggers monitoring, adjustment, and learning processes (as does failed capture following an accept decision). Rejecting a prey item is passive, maintains the status quo (that is, continuing to search), and, during the subsequent search, returns the decision-maker to outwardly-oriented searching (formally, exploratory) mode.

\section{Insights into self-control from the accept/reject framework}

One important example of the way the accept/reject framing matters is the performance of animal decision-makers in intertemporal choice (a.k.a. delay discounting) tasks. Such tasks, in which animals choose between delayed large rewards and immediate small rewards, are a mainstay of the psychology and neuroscience of self-control (reviewed in Hayden 2015). Animals generally appear impulsive, meaning they prefer a reward offered sooner even if it is less profitable. This observation is difficult to reconcile with evolutionary theory because it is highly maladaptive in the long run. However, intertemporal choice tasks, structured as binary choice tasks, will lead a decision-maker evolved to making accept-reject decisions to misattribute delays appear impulsive (Stephens et al., 2004; Bateson and Kacelnik, 1996). Indeed, in foraging tasks with a time component, ostensibly impulsive animals are almost perfectly patient (Blanchard and Hayden, 2015; Stephens and Anderson, 2001; Pearson et al., 2010; Carter and Redish, 2016). 
Another important feature of foraging decisions absent from standard economic ones is the requirement for persistence in pursuing or handling a prey item after the decision itself it made. The neural mechanisms of persistence are just beginning to be understood (Hillman and Bilkey, 2013; McGuire and Kable, 2015; Wittmann, 2016; Rudebeck, 2006). One key ingredient in many persistence decisions is the need to maintain an ongoing representation of the value of the prey and to update that value continuously as the receipt of the prey gets closer in time (McGuire and Kable, 2015; Blanchard et al., 2015). Failures of this value updating process may help to explain failures of self-control, and treatments that modify this representation may help improve self-control.

\section{How accept-reject decisions are implemented}

The key decision variable in accept-reject decision is profitability: the gain weighed against the cost of the item, including opportunity costs (Stephens and Krebs, 1986). Profitability is compared to a threshold, the average value of the environment (the analogous term in baseball statistics is the Mendoza Line). The most straightforward way to implement an accept-reject decision is to maintain a (dynamic) representation of the profitability of the foreground option and a (stable) representation of the profitability of the background and to compare them (Kolling et al., 2012; Wittmann et al., 2016; Hayden et al., 2011). Control systems in the brain then can modulate these representations regulate the threshold for accepting a presented option (Kolling et al, 2014; Hayden et al., 2011; Steiner and Redish, 2015).

Once an item is attended, accepting the option may be a type of default action; rejecting it would then be an alternative. If so, this framing would introduce an asymmetry into binary choice. That asymmetry should be visible in the brain, and indeed it is: the two option types are associated with activation in the ventromedial prefrontal cortex (vmPFC) and dorsal anterior cingulate cortex (dACC), for default and alternative, respectively (Boorman et al., 2013; Kolling et al., 2012). Presumably then choice is determined by competition processes between these two systems. The role of dACC in encoding the value of the alternative is also consistent with recordings of single neurons there, which show encoding of the rejected value on reject trials and of the delay - which corresponds to the opportunity cost of the accept decision - on accept trials (Blanchard and Hayden, 2014). And in a patch-leaving task, in which the decision to reject an option builds over several trials, responses of dACC neurons gradually increase as the value of rejection rises (Hayden et al., 2011). These discoveries about dACC offer a synoptic account of dACC function that was not available from standard conflict and comparator models based on conventional (non-foraging) tasks (Kolling et al., 2016; Heilbronner and Hayden, 2016).

\section{Are ostensibly binary choices really paired accept-reject choices?}

Binary choice is the very core of microeconomics and understanding its neural basis is a central goal of neuroeconomics. Given the importance of accept/reject decisions in foraging, however, some scholars have argued that the binary choice is at least somewhat unnatural and in some cases an artificial laboratory construct (Kacelnik et al., 2011; Stephens et al., 2004). A forager whose brain is evolved for single encounters may treat the binary choice as two simultaneous accept/reject decisions. Key evidence for this decision mechanism comes from measures of reaction times and choice probabilities (Shapiro et al, 2008; Freidin et al, 2009; Vasconcelos et 
al, 2010; Pirrone et al, 2016). An implication of these results is that binary choice is better described as a paired race to threshold than a drift diffusion between two bounds.

Another psychological limitation on binary choice is the limited capacity of attention: we cannot bind abstract features (like value) to objects (offers) in the absence of attention, which is generally limited to a single spotlight (Treisman and Gelade, 1980). In a standard visual task with two spatially separate options, the spotlight of attention likely follows the locus of gaze or, sometimes, covert attention (Krajbich et al, 2011). In more abstract situations, such as when choosing between two possible options that are out of view (e.g. a monkey choosing which of two distant orchards to forage in), the locus of attention likely shifts in a more abstract manner, but still serially. Thus, it seems likely that when options are presented simultaneously, they are nonetheless evaluated and compared serially. Key evidence for this idea comes from a study that recorded ensemble activity in orbitofrontal cortex (OFC) in a simultaneous choice task (Rich and Wallis, 2016). Neuronal ensembles rapidly oscillated between two states corresponding to the two possible options, presumably tracking the focus of attention. Further evidence comes from the fact that, when attention is artificially controlled (by controlling gaze), ventromedial prefrontal cortex (vmPFC) and OFC preferentially track the values of attended offers (Lim et al, 2011, Strait et al, 2014; McGinty et al., 2016; Blanchard et al., 2015; Xie, Nie, and Yang, 2017).

\section{How can comparison occur in serial choice models?}

If attention oscillates between single offers, and the brain signals the value of the attended offer only, how can a comparison occur? One possibility is that the brain computes the relative, not absolute, value of the attended offer (that is, the value difference or quotient). This relative value can be seen as a normalized representation of the value of the offer, but is sufficient to guide choice: if the difference is greater than zero, or if the quotient is greater than 1 , the attended option can be selected. There is evidence that value representation in vmPFC is relative (Strait et al, 2014, Lim et al, 2011) and may be relative in other areas as well (e.g. Hunt et al., 2014; Strait et al, 2015; Hunt et al, 2012; Klein-Flugge et al, 2016; Padoa-Schioppa, 2009). To implement choice, then, such normalized value representations must be subject to some downstream (or distributed) comparison-to-threshold process.

During serial shifts of attention, what is occurring during each epoch of sustained attention on one option? It seems likely that the brain is gradually accumulating evidence in favor of or against selecting that option (Krajbich et al, 2011; Pisauro et al, 2017). That evidence is presumably stochastic, because it reflects the output of multiple noisy channels. It seems likely that at least some of that sampling corresponds drawing recollections of stimulus and action value mappings from memory (Shadlen and Shohamy, 2016; Ludvig et al, 2014). These are then fed into one or more value buffers and compared to a threshold.

\section{Foraging suggests a unification of economic and stopping decisions}

An accept/reject decision is a choice between actively changing the status quo or passively maintaining it and continuing to search; accepting involves performing a planned or primed motoric response; rejection involves withholding it. In other words, an accept/reject decision has much in common with a stopping decision. And binary choice, by extension, has much in common with a pair of interacting stopping decisions. This speculation, if true, is important because 
the neural mechanisms of stopping are relatively well understood, and applying this understanding to economic decisions could rapidly advance the neuroscience of economic choice (Logan et al., 2015; Aron et al, 2004; Hampshire and Sharp, 2015). Indeed, if economic choice ultimately boils down to stopping, there is an opportunity for a "grand unified theory" unifying the two types of decisions.

There is some tentative evidence that the neural circuitry involved in stopping is overlapping with the circuitry involved in economic decisions. The motor and premotor cortex, for example, have clearly defined roles in stopping decisions, and also have important and complementary roles in economic choices (Cisek and Kalaska, 2010; Cisek, 2012). More broadly, at least some evidence supports the idea that stopping is a distributed process that reflects activity of much of the prefrontal cortex (among other regions, Hampshire and Sharp, 2015); similar arguments have been made for economic choice (Cisek, 2012; Hunt and Hayden, 2017). In any case, future work on the relationship between stopping and choice is needed. Progress in this area promises to help shed light on important debates, such as how economic choice relates to selfcontrol (Berkman et al., 2017; Shenhav, 2017).

\section{Value as tentative commitment to a decision}

Value is a construct that is convenient in economic models, but may not be explicitly computed; evidence that it is realized in the brain is equivocal (Hunt and Hayden, 2017; Vlaev et al., 2011). The brain has not evolved to compute value and then use that to drive choice; it has evolved to drive choice (Cisek and Kalaska, 2010). Indeed, a reasonable null hypothesis would be that the brain, as an evolved system, performs a gradual rotation from an input to an output space without a special amodal value representation in a middle layer. Such a rotation would lead to sensorimotor information - that is, the details of the positions of offers and actions leading to them, in ostensibly motor areas. Recent evidence supports the idea that such signals are indeed observed throughout the reward system (Strait et al, 2016; McGinty et al., 2016; Bryden and Roesch, 2015; Luk and Wallis, 2013; Cisek, 2012). What we call value, then, may really a tentative commitment to accepting an offer, or, more abstractly, to a proposition (cf. Shadlen et al, 2008). In early layers, that proposition may be toward identifying the stimulus, in middle layers, it may be towards signals that can influence the action or the goal, and in later layers it may be towards the action associated with choosing it (Cisek, 2012; Hunt and Hayden, 2017; Hunt et al, 2014; Cisek and Kalaska, 2010). Future studies will be necessary to test this idea; such studies are most likely to be informative if they are ethologically relevant, that is, if they embed the decision-maker in as natural an environment as possible (Pearson et al., 2014). 


\section{REFERENCES}

1. Aron, A. R., Robbins, T. W., \& Poldrack, R. A. (2004). Inhibition and the right inferior frontal cortex. Trends in Cognitive Sciences 8(4), 170-177.

2. Bateson, M., \& Kacelnik, A. (1996). Rate currencies and the foraging starling: The fallacy of the averages revisited. Behavioral Ecology 7:341-352.

3. • Berkman, E. T., Hutcherson, C. A., Livingston, J. L., Kahn, L. E., \& Inzlicht, M. (2017). Self Control as Value-Based Choice. Current Directions in Psychological Science. In press.

4. Blanchard T. C. \& Hayden, B. Y. (2014). Neurons in dorsal anterior cingulate cortex signal postdecisional variables in a foraging task. Journal of Neuroscience 34:646-55

5. - Blanchard, T. C., \& Hayden, B. Y. (2015). Monkeys are more patient in a foraging task than in a standard intertemporal choice task. PLoS ONE 10:e0117057. doi:10.1371/journal.pone.0117057

6. Blanchard TC, Hayden BY, and Bromberg-Martin E (2015). Orbitofrontal cortex uses distinct codes for different choice atributes in choices motivated by curiosity. Neuron 85:602-614.

7. •• Blanchard, T. C., Strait, C. E., \& Hayden, B. Y. (2015). Ramping ensemble activity in dorsal anterior cingulate neurons during persistent commitment to a decision. Journal of Neurophysiology, 114(4): 2439-2449.

Monkeys performed a diet-selection task (i.e. an accept-reject task with delayed rewards) that required persistence after the reward was chosen to obtain it. Firing rates of neurons in dACC continuously encoded the anticipated value, but used a gradually changing format to do so as the reward approached.

8. Boorman ED, Rushworth MF, Behrens TE. 2013. Ventromedial prefrontal and anterior cingulate cortex adopt choice and default reference frames during sequential multi-alternative choice. Journal of Neuroscience 33:2242-53.

9. •• Bryden, D. W. and Roesch, M. R. (2015) Executive control signals in orbitofrontal cortex during response inhibition. Journal of Neuroscience 35(9):3903-3914.

A good deal of evidence supports a role for OFC in inhibition, but unit activity has seldom been recorded during a stop signal task. In rats performing the task, neurons strongly encoded the direction and speed of movement, suggesting that OFC plays a supervisory or executive role in inhibition.

10. Carter, E. C. And Redish, A. D. (2016). Rats value time differently on equivalent foraging and delay discounting tasks. Journal of Experimental Psychology: General 145(9) 1093-1101.

11. Cisek, P., \& Kalaska, J. F. (2010). Neural mechanisms for interacting with a world full of action choices. Annual Review of Neuroscience 33:269-298.

12. - Cisek, P. (2012). Making decisions through a distributed consensus. Current Opinion in Neurobiology 22(6):927-936.

13. Freidin, E., Aw, J., \& Kacelnik, A. (2009). Sequential and simultaneous choices: testing the diet selection and sequential choice models. Behavioural processes 80(3):218-223. 
14. Hampshire, A., \& Sharp, D. J. (2015). Contrasting network and modular perspectives on inhibitory control. Trends in Cognitive Sciences 19(8):445-452.

15. Hayden, B. Y., Pearson, J. M., \& Platt, M. L. (2011). Neuronal basis of sequential foraging decisions in a patchy environment. Nature Neuroscience 14(7):933-939.

16. Hayden B. Y. (2016). Time discounting and time preference in animals: a critical review. Psychonomic Bulletin and Review 23:39-53.

17. Heilbronner S. R. and Hayden B. Y. (2016). Dorsal anterior cingulate cortex: a bottom up view. Annual Review in Neuroscience 39: 149-170.

18. Hillman K. L., Bilkey D. K. 2010. Neurons in the rat anterior cingulate cortex dynamically encode cost-benefit in a spatial decision-making task. Journal of Neuroscience 30:7705-13.

19. • Hunt L. T., Dolan R. J., Behrens T. E. (2014) Hierarchical competitions subserving multiattribute choice. Nature Neuroscience 17:1613-1622.

20. • Hunt, L. T. \& Hayden, B. Y. (2017) A distributed, hierarchical and recurrent framework for reward-based choice. Nature Reviews Neuroscience 18:172-182.

21. Hunt, L. T., Kolling, N., Soltani, A., Woolrich, M. W., Rushworth, M. F., and Behrens, T. E. (2012). Mechanisms underlying cortical activity during value-guided choice. Nature Neuroscience 15:470-476.

22. Kacelnik, A., Vasconcelos, M., Monteiro, T., and Aw, J. (2011). Darwin's “tug-of- war" vs. starlings' "horse-racing": how adaptations for sequential encounters drive simultaneous choice. Behavioral Ecology and Sociobiology 65:547-558.

23. Klein-Flügge, M. C., Kennerley, S. W., Friston, K., and Bestmann, S.. (2016). Neural signatures of value comparison in human cingulate cortex during decisions requiring an effort-reward trade-off. Journal of Neuroscience 36(39):10002-10015.

24. • Kolling, N., Wittmann, M. \& Rushworth, M.F. (2014) Multiple neural mechanisms of decision making and their competition under changing risk pressure. Neuron 81:1190-1202.

25. Kolling, N., Wittmann, M. K., Behrens, T. E. J., Boorman, E. D., Mars, Rogier B \& Rushworth, M. F. S. (2016). Value, search, persistence and model updating in anterior cingulate cortex. Nature Neuroscience 19(10):1280-1285.

26. Kolling, N., Behrens, T.E.J., Mars, R.B., \& Rushworth, M.F.S. (2012) Neural mechanisms of foraging. Science 336:95-98.

27. Krajbich, I., Armel, C., \& Rangel, A. (2010). Visual fixations and the computation and comparison of value in simple choice. Nature neuroscience 13(10):1292-1298.

28. Lim, S. L., O'Doherty, J. P., \& Rangel, A. (2011). The decision value computations in the vmPFC and striatum use a relative value code that is guided by visual attention. Journal of Neuroscience 31(37) 13214-13223.

29. Logan, G. D., Yamaguchi, M., Schall, J. D., \& Palmeri, T. J. (2015). Inhibitory control in mind and brain 2.0: Blocked-input models of saccadic countermanding. Psychological Review 122:115-147. 
30. • Ludvig, E. A., Madan, C. R., \& Spetch, M. L. (2015). Priming memories of past wins induces risk seeking. Journal of Experimental Psychology: General 144(1) 24-29.

31. Luk C-H., Wallis J. D. 2013. Choice coding in frontal cortex during stimulus-guided or action-guided decision-making. Journal of Neuroscience 33:1864-71.

32. • McGinty, V. B., Rangel, A., Newsome, W. T., 2016. Orbitofrontal Cortex Value Signals Depend on Fixation Location during Free Viewing. Neuron 90:1299-311.

33. • McGuire J. T., Kable J. W. Medial prefrontal cortical activity reflects dynamic re-evaluation during voluntary persistence. Nature Neuroscience 18: 760-766, 2015.

Human subjects performed delay of gratification tasks with different parameters that differentially rewarded persistence. Subjects calibrated their persistence and neural correlates of value followed. These results highlight the importance of rational valuation processes, over non-valuerelated executive processes in governing persistence.

34. Padoa-Schioppa, C. (2009). Range adapting representation of economic value in the orbitofrontal cortex. Journal of Neuroscience 29(44) 14004-14014.

35. • Passingham, R., \& Wise, S. (2012). The neurobiology of the prefrontal cortex: Anatomy, evolution, and the origin of insight. Oxford, UK: Oxford University Press.

36. Pearson, J. M., Hayden, B. Y., \& Platt, M. L. (2010). Explicit information reduces discounting behavior in monkeys. Frontiers in Psychology, 1, 237. doi:10.3389/fpsyg.2010.00237

37. • Pearson J. M., Watson K. K., Platt M. L.: Decision making: the neuroethological turn. Neuron 2014, 82:950-965.

38. Pisauro, M. A., Fouragnan, E., Retzler, C., and Philiastides, M. G. (2017). Neural correlates of evidence accumulation during value-based decisions revealed via simultaneous EEG-fMRI. Nature Communications. 8:15808 DOI: 10.1038/ncomms15808 | www.nature.com/naturecommunications

39. Pirrone A, Azab H, Hayden BY, Stafford T, Marshall JAR: Evidence for the speed-value trade-off: human and monkey decision making is magnitude sensitive. Decision 2017 . in press http:// dx.doi.org/10.1037/dec0000075. 2

40. •• Rich, E. L., \& Wallis, J. D. (2016). Decoding subjective decisions from orbitofrontal cortex. Nature Neuroscience 19(7):973-980.

Macaques performed a binary choice task while electrode arrays in OFC monitored ensemble activity. During deliberation, ensemble state alternated between two discrete states, corresponding to the two options. These results may correspond to the shifting spotlight of attention during deliberation.

41. Rudebeck, P. H., Walton, M. E., Smyth, A. N., Bannerman, D. M., \& Rushworth, M. F. S. (2006). Separate neural pathways process different decision costs. Nature Neuroscience 9(9) 1161-1168.

42. • Shadlen, M. N. \& Shohamy, D. Decision making and sequential sampling from memory. Neuron 90, 927-939 (2016).

A good deal of evidence supports the idea that economic decisions can be modeled as sequential sampling processes, but what gets sampled? This Perspective argues that it is samples of 
value in memory. It lays out a helpful neuroanatomical hypothesis, which should guide future tests of the hypothesis.

43. Shadlen, M., Kiani, R., Hanks, T., and Churchland, A.K. (2008). Neurobiology of decision making: an intentional framework. In Better than Conscious? Decision Making, the Human Mind, and Implications for Institutions, C. Engel and W. Singer, eds. (Cambridge: MIT Press), pp. 71-102.

44. Shapiro, M. S., Siller, S., and Kacelnik, A. (2008). Simultaneous and sequential choice as a function of reward delay and magnitude: normative, descriptive and process-based models tested in the European starling (Sturnus vulgaris). Journal of Experimental Psychology: Animal Behavioral Processes 34:75-93.

45. Shenhav, A. (2017). The perils of losing control: why self-control is not just another valuebased decision. Psychological Inquiry. In press.

46. •• Steiner, A.P., Redish, A.D., (2014). Behavioral and neurophysiological correlates of regret in rat decision-making on a neuroeconomic task. Nature Neuroscience 17:995-1002. doi:10.1038/nn. 3740

Rats performed a diet selection task (the Restaurant Row Task). Following decisions to skip a low-cost choice and encountering an inferior high-cost option, rats demonstrated regret-like behavior and their OFC and VS encoded the value of the foregone option. These results provide an important window into the complex psychology underlying foraging decisions.

47. Stephens, D. W., \& Krebs, J. R. (1986). Foraging theory. Princeton University Press.

48. Stephens, D. W., Kerr, B., \& Fernández-Juricic, E. (2004). Impulsiveness without discounting: The ecological rationality hypothesis. Proceedings of the Royal Society B, 271, 2459-2465. doi: 10.1098/rspb.2004.2871

49. Stephens, D. W., \& Anderson, D. (2001). The adaptive value of preference for immediacy: When shortsighted rules have farsighted consequences. Behavioral Ecology, 12, 330-339.

50. Strait, C.E., Blanchard, T.C., Hayden, B.Y. (2014). Reward Value Comparison via Mutual Inhibition in Ventromedial Prefrontal Cortex. Neuron 82:1357-1366.

51. Strait, C. E., Sleezer, B. J., \& Hayden, B. Y. (2015). Signatures of value comparison in ventral striatum neurons. PLoS Biol, 13(6), e1002173.

52. Strait, C. E., Sleezer, B. J., Azab, H., Castagno, M., and Hayden, B. Y. (2016). Neuronal selectivity for spatial positions of offers and choices in five reward regions. J. Neurophysiol. 115, 10981111.

53. Treisman, A. M., \& Gelade, G. (1980). A feature-integration theory of attention. Cognitive psychology, 12(1), 97-136.

54. Vlaev, I., Chater, N., Stewart, N., and Brown, G. D. A. (2011). Does the brain calculate value? Trends in Cognitive Science 15: 546-554.

55. Vasconcelos M, Monteiro T, Aw J, Kacelnik A (2010) Choice in multi-alternative environments: a trial-by-trial implementation of the sequential choice model. Behavioral Processes 84:435439 
56. •• Wittmann, M. K., Kolling, N., Akaishi, R., Chau, B. K. H., Brown, J. W., Neilsen, N., and Rushworth, M. F. S. (2016) Predictive decision making driven by multiple time-linked reward representations in the anterior cingulate cortex. Nature Communications 7:12327.

Human subjects performed a computerized foraging task based on the patch-leaving paradigm, with a patch-richness that required comparing recent and further past yields to estimate trends. Different components of the computation were modeled with reinforcement learning and corresponded to specific anatomical regions.

57. Xie, Y., Nie, C., and Yang, T. (2017) Covert shift of attention modulates the value encoding in the orbitofrontal cortex. bioRxiv. doi: https://doi.org/10.1101/181784 


\section{Figures}

Figure 1. Animals in natural environments, such as this gorilla at the Karisoke Research Center in Rwanda, generally encounter prey one at a time. Their decision-making strategies are molded by those encounters and are centered on accept-reject decisions. (Photo credit: Jessica Cantlon).

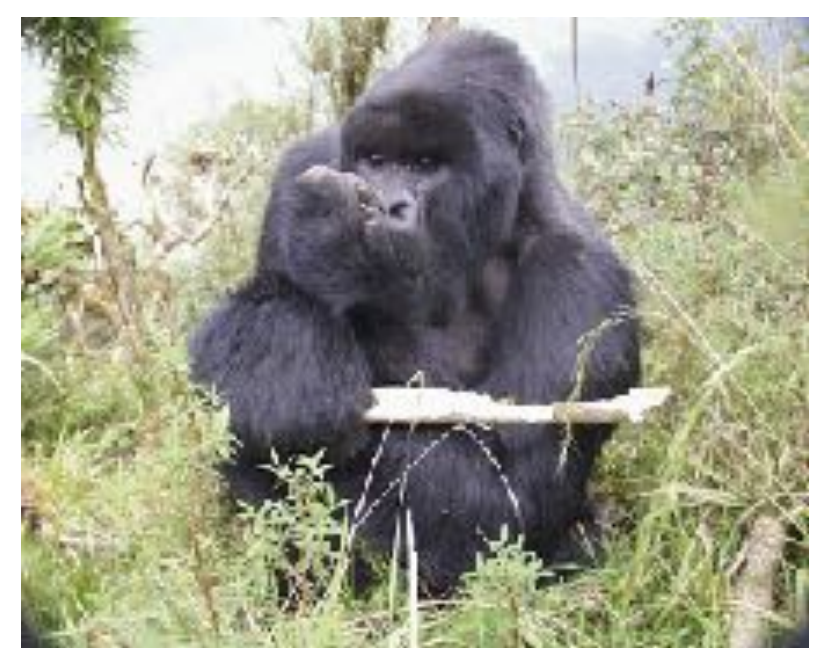

Figure 2. Animals in the laboratory, such as this baboon at the Seneca Park Zoo, are often faced with binary decisions. Some research suggests that those decisions are made as interleaved accept-reject decisions of each option. (Photo credit: Jessica Cantlon).

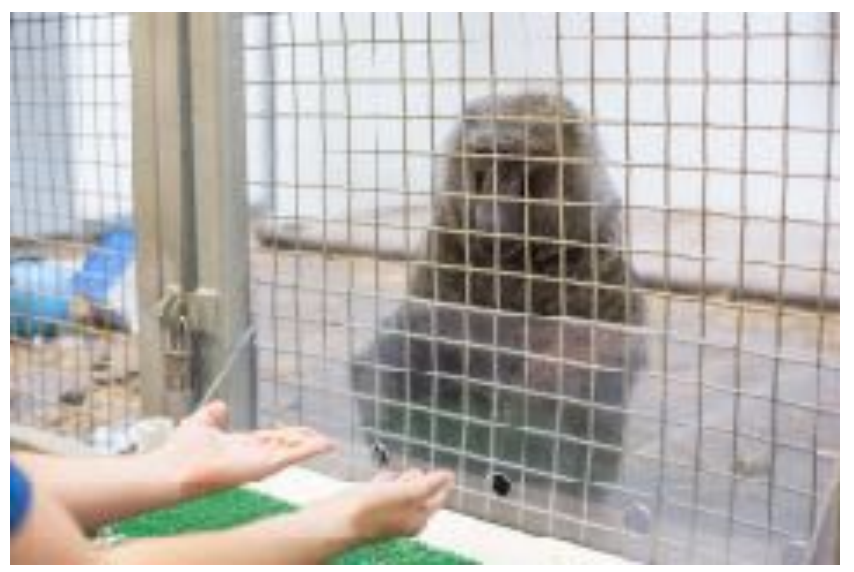

